\title{
WDM Coding for High-Capacity Lightwave Systems
}

\author{
Shou-Kuo Shao and Ming-Seng Kao
}

\begin{abstract}
An interchannel parallel coding scheme in the wavelength domain-the WDM coding system-is proposed. The system differs from the usual serial coding systems and provides many advantages. First, data channels are completely unaltered in the coding process, rendering it very suitable for practical lightwave systems with standard bit rate. Second, parallel encoding/decoding systems are simpler than those of serial coding systems, being easier to be implemented in high-speed optical systems. Third, compared with serial coding, WDM coding is able to reduce heavily the number of encoding/decoding pairs. For example, a $(15,11)$ Hamming coded WDM system reduces the number from $12 \times 11=132$ to 1 at the line rate of STS-12. Fourth, the WDM coding system could offer infinite coding gain in dispersion-limited lightwave systems. Finally, WDM coding systems could correct single-channel burst error. The system performance was evaluated and the system limitation imposed by bit-skew among wavelength channels was analyzed. The results indicated that a 15-channel Hamming coded WDM system can reduce the uncoded BER from $10^{-9}$ to $3 \times 10^{-17}$ and the distance limitation imposed by bit-skew is $250 \mathrm{~km}$ if a dispersion-shifted fiber is used and a channel span of $\mathbf{3 0} \mathrm{nm}$ is assumed
\end{abstract}

\section{INTRODUCTION}

I $\mathrm{N}$ general, optical channel capacity can be classified in spatial and temporal domains [1]. The spatial channel capacity in bits-per-unit spatial area can be defined as $C \triangleq \lim _{S \rightarrow \infty}\left[\log _{2} N(S) / S\right]$ bits/unit area, where $S$ is the spatial plane and $N(S)$ is the total combinations of the cells. When the optical channel is reduced to a single resolution cell, the capacity of the channel is characterized as $C \triangleq \lim _{T \rightarrow \infty}\left[\log _{2} N(T) / T\right]$ bits/s, where $N(T)$ is the total possible distinct combination of quantized signal levels during a long period of time $T$. This is the well-known formula of channel capacity derived by Shannon. Shannon's second theorem tells us that if a source has an information rate less than the channel capacity, there exists an encoding procedure such that the source output can be transmitted over the channel with an arbitrarily small probability of error [2]. Shannon's theorem predicts essentially error-free transmission in the presence of noise; namely, reliable communication is achievable when long codewords are used [3].

In lightwave communications, coding has been proposed for several purposes [4]-[6]. However, they are rarely used in practical lightwave systems because 1) coding in general changes system bit rate that is unacceptable for practical systems with well-specified bit rate, 2) the required signal processing in encoding/decoding processes is difficult in highspeed lightwave systems, and 3) coding gain is small in

Manuscript received December 25, 1992; revised February 23, 1993.

The authors are with the Department of Communication Engineering and the Center for Telecommunications Research, National Chiao Tung University, Hsinchu, Taiwan, Republic of China.

IEEE Log Number 9210175. power-limited lightwave systems. Nevertheless, some practical applications of forward error correction (FEC) code at the rate of DS-3 and STS-1 are presented by Grover [7] and Grover and Moore [8]. In their coding systems, they smartly put paritycheck bits in some unused stuff bits of DS-3 or unassigned overhead fields of STS-1 in order not to affect system bit rate. Moreover, to avoid high-speed signal processing, they definitely implement the FEC coding at low-speed tributaries of high-speed systems. For instance, they implement FEC at STS-1 level and multiplex N STS-1 channels to form an STS-N high-speed system by $50-\mathrm{MHz}$ CMOS gate-arrays. Fig. 1 shows the architecture of FEC implementation for an STS-12 transmission system designed by Grover. Each STS-1 tributary is first encoded at its own FEC encoder and then multiplexed together to be an STS-12 signal. In the receiving end, the signal is demultiplexed into 12 encoded STS-1 signals and then decoded by FEC decoders to recover the primitive STS-1 data. In the coding system, we need 12 encoding/decoding pairs operating at STS-1 level to allow for complicated signal processing involved in putting parity-check bits into unassigned overhead fields.

Grover demonstrated the astonishing power of FEC in dispersion-limited lightwave systems where a powerindependent BER floor appears resulting from impairments such as mode partition noise (MPN), mode hopping, lasertransient chirping, and reflection-induced noise. Although the coding gain for single-error FEC in power-limited systems is estimated to be only $1.5 \mathrm{~dB}, \mathrm{FEC}$ can have infinite coding gain in dispersion-limited operation. Indeed, FEC allows: 1) increase of effective yield of lasers, 2) relaxation or elimination of optical source isolation, and 3) exploitation of conventional fibers in long-haul high-speed lightwave systems where BER floors preclude their use [7]. However, there are some inherent problems in Grover's design. First, when the transmission capacity is large, the number of FEC encoder/decoder pairs will be large-we need 12 encoder/decoder pairs for an STS-12 system. Still, the number will be very large for a high-capacity wavelength division multiplexing (WDM) lightwave system. Second, placing parity-check bits in unassigned overhead fields requires accurate timing and complicated signal processing, rendering it difficult to operate the FEC at a rate higher than STS- 1 .

A single-mode fiber provides enormous bandwidth around the $1.55-\mu \mathrm{m}$ low-loss wavelength region. On the one hand, the bandwidth can be used to achieve very high capacity information transmission through the WDM technique [9]. On the other hand, from Shannon's theorem, the abundant bandwidth can be used for coding purpose to achieve nearly error-free transmission. In this paper, we propose the WDM coding scheme to reach a reliable high-capacity WDM system. 


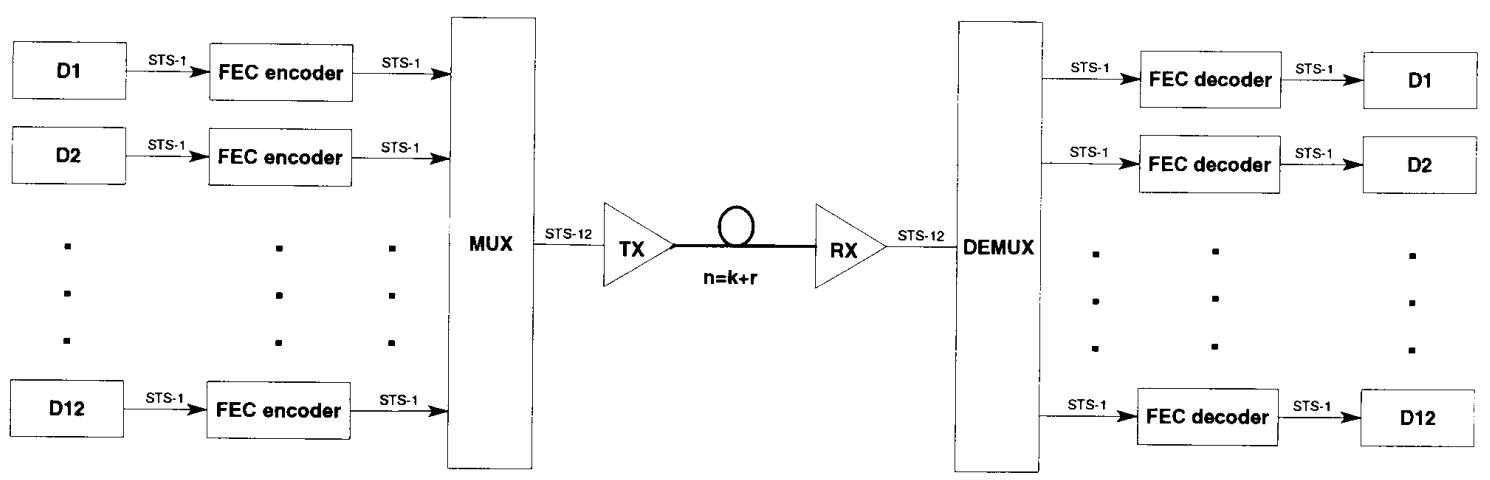

Fig. 1. Architecture of high-speed transmission link with FEC in low-speed tributary interfaces.

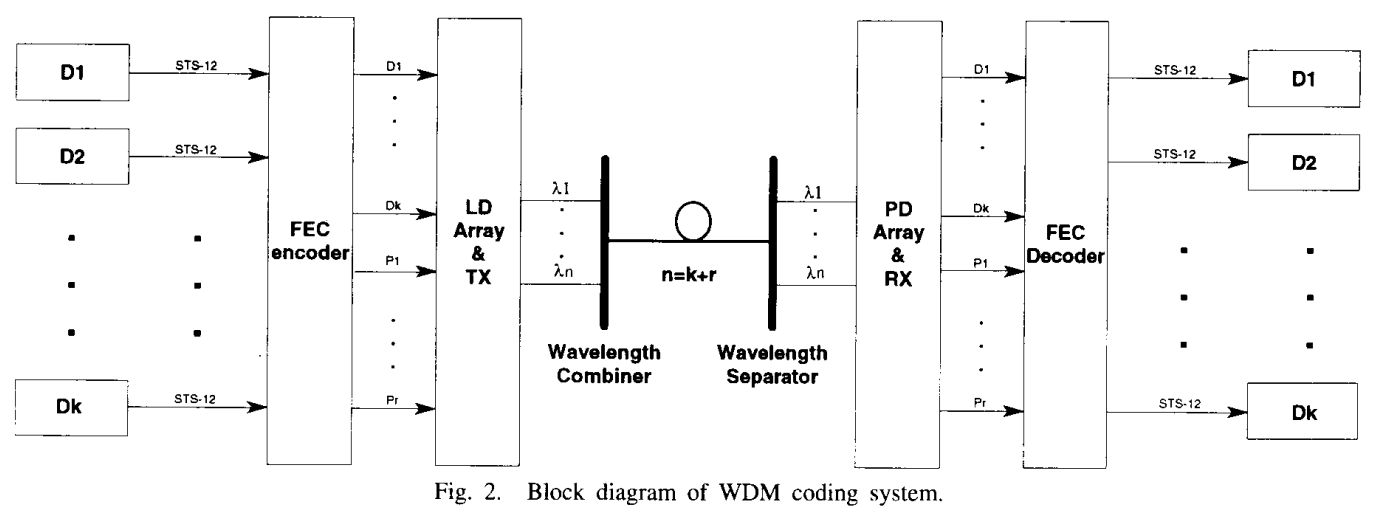

The WDM coding system is motivated by two concerns: 1) utilizing the fiber bandwidth and Shannon's theorem to approach nearly error-free transmission, and 2) introducing parallel coding to WDM systems to achieve a simple and high-speed coding system. The architecture of the proposed WDM coding system is given in Fig. 2. $k$ independent data sequences $\left(D_{1}, \cdots, D_{k}\right)$, each operating at a STS-12 rate, are encoded in parallel by an FEC encoder to output codeword sequences $\left(D_{1}, D_{2}, \cdots, D_{k}, P_{1}, \cdots, P_{r}\right)$. Here the original data sequences $\left(D_{1}, \cdots, D_{k}\right)$ are unaffected by the encoder, and the generated parity-check sequences $\left(P_{1}, \cdots, P_{r}\right)$ have the same rate as the data sequences. Thus we have an $(n, k)$ coding system where $n=k+r$ is the codeword length. Unlike the usual serial coding, the present coding is performed in parallel by taking one bit out of each data sequence to form a length- $k$ data word, then by using the FEC encoder to produce $r$ parity-check bits. The $r$ parity-check bits are carried by $r$ parity-check channels. The $n$ codeword sequences then modulate an LD array consisting of $n$ different wavelength lasers $\left(\lambda_{1}, \cdots, \lambda_{n}\right)$ to form an $n$-channel WDM transmission. At the receiving end, the $n$ wavelength channels are separately received and the codeword sequences are decoded by an FEC decoder to retrieve original data sequences.

The salient features of the WDM coding system include the following: 1) WDM coding systems put parity-check bits at different wavelength channels. This leaves data channels unaffected by the coding process-that is, the system bit rate is unchanged; 2) By placing parity-check bits in additional wave- length channels, its encoding/decoding process is much simpler than the serial coding system which places parity-check bits in unassigned overhead fields. This allows for high-speed encoding/decoding (STS-12 instead of STS-1); and 3) Parallel coding can reduce heavily the number of encoder/decoder pairs. For example, a $(15,11)$ WDM Hamming coded system operating at STS-12 level needs only one encoder/decoder pair, whereas the number of encoder/decoder pairs of Grover's design will be $11 \times 12=132.4$ ) As is indicated later, WDM coding systems can correct one channel burst error.

The paper is organized as follows: We first describe the code and system configuration of the WDM coding system in Section II. Next we analyze the system performance and limitations in Section III. We then discuss important characteristics of WDM coding systems in Section IV. Finally, we present our conclusions in Section V.

\section{SySTEM DESCRIPTION}

As illustrated in Fig. 2, $k$ data channels are fed into the FEC encoder to produce $n$ codeword channels. In order to understand the coding strategy being used, we discuss the coding scheme first and then talk about TX and RX configurations.

\section{A. Coding Scheme}

In the proposed WDM coding system, we adopt the simplest, most well known, and truly parallel coding scheme-the Hamming code [10], [11]. The reasons why we choose Hamming code are summarized here: 


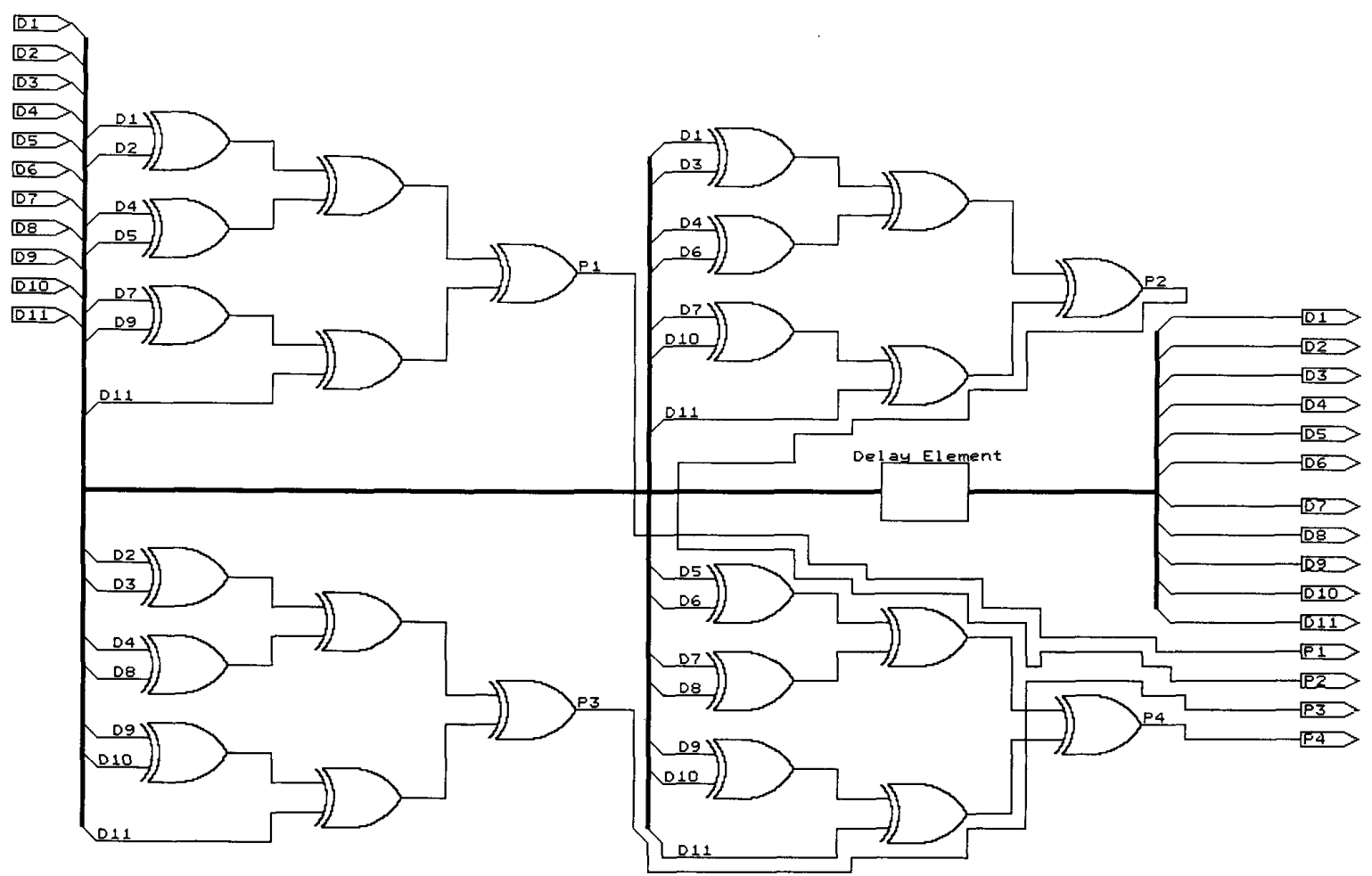

Fig. 3. Encoder circuit of a $(15,11)$ SEC Hamming code.

1. The BER in ordinary lightwave systems is usually less than $10^{-9}$. With such a low BER, it is unnecessary to use a powerful FEC code with complex encoding/decoding processes. The single-error-correcting capability of Hamming code has been enough to reduce the BER to $O\left(P_{e}^{2}\right)$, where $P_{e}$ is the BER without coding.

2. In the WDM coding system, we make use of wavelength multiplexing to construct a parallel coding for high-speed long-haul links. One critical factor that prohibits the high-speed coding is the electronics speed. Therefore, we prefer the simplest coding scheme feasible by high-speed electronics.

3. Error extension due to miscorrection may affect severely interchannel WDM coding systems. To avoid this impact, we use the Hamming code with small error extension.

4. Hamming code provides flexible codeword length which is desirable in the WDM coding system whose channel number may be varied.

5. In the WDM coding system, Hamming code keeps the advantage that data channels remain unchanged after encoding - that is, we just add a few wavelength channels to carry parity-check bits without affecting the original data channels. This feature is desirable since it simplifies signal processing.

From the above considerations, Hamming code having a parallel encoding/decoding process is appropriate for the WDM coding system. Hamming code is characterized by the length of the codeword and the length of the data segment, which is expressed in the conventional $(n, k)$ notation, and which is specified directly by its parity-check matrix $\boldsymbol{H}$. Take a $(15,11)$ Hamming code, for instance:

data length, $k: k=11$

number of parities, $r: r=4$

block length of codeword, $n: n=2^{r}-1=15$

minimum distance $d_{\text {min }}: d_{\min }=3$

and

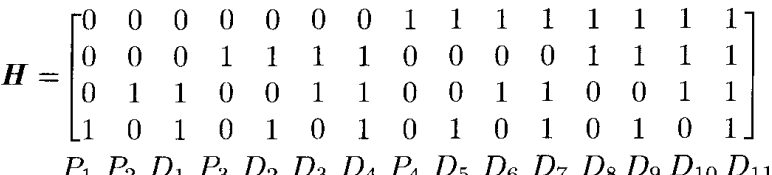

From the parity-check matrix, we can calculate the paritycheck equations, which constitute the encoder circuit in the transmitting end, and the syndrome equations, which constitute the decoder circuit in the receiving end.

\section{B. TX Configuration}

As shown in Fig. 2, $k$ data channels are encoded first in the FEC encoder at the STS-12 line rate to generate $r$ parity-check channel $\left(P_{1}, \cdots, P_{r}\right)$, each still at the STS-12 line rate. The parity-check channels are obtained simply by computing by the "parity equations" given in [10]. Then the $n$ signals modulate the LD array to construct an $n$-channel WDM transmission. The encoder circuit of a $(15,11)$ Hamming code based on parity equations for single-error correction (SEC) is shown in Fig. 3 where each parity-check bit has a modulo-2 sum of 


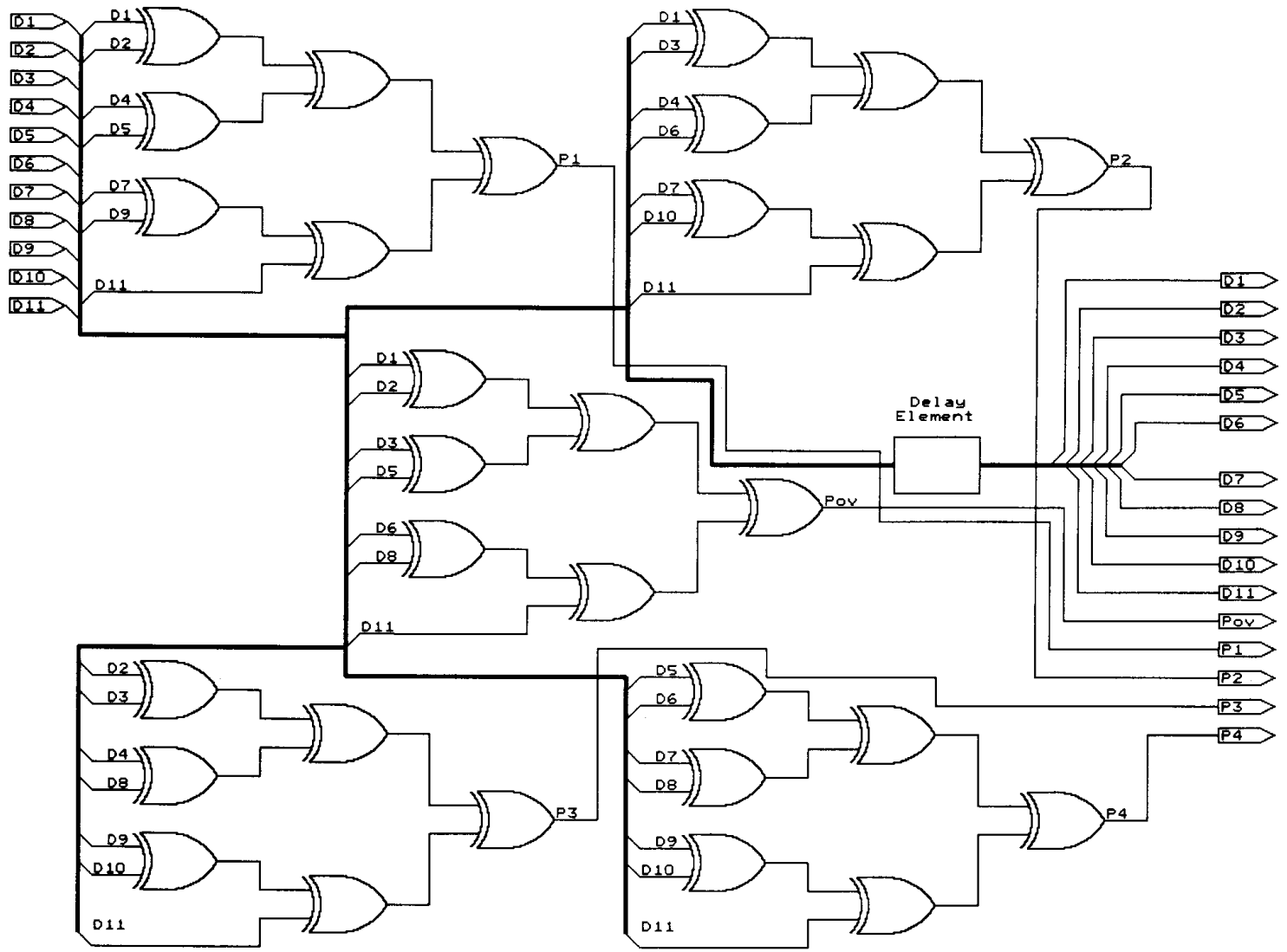

Fig. 4. Encoder circuit of a $(16,11)$ modified SEC-DED Hamming code.

7 data channels and the data channels are delayed in order to synchronize with parity-check channels. Fig. 4 shows the encoder circuit of a $(16,11)$ modified Hamming code for single-error correction and double-error detection (SEC-DED). These circuits consist of simple exclusive-OR gates only which can be implemented easily at the rate of STS-1.

\section{C. $R X$ Configuration}

Fig. 5 shows the detail receiver architecture of the WDM coding ksystem. After the wavelength splitter, the $n$ signals are sent to the PD array and fed to $n$ decision and timing recovery circuits. Due to fiber dispersion, the group velocity of each wavelength channel is different. Consequently, the $n$ channel signals, being simultaneously transmitted, may arrive at the receiving end at a different time. This is the bit-skew phenomenon, a unique property of parallel transmission [12]. As a result, each channel signal arrives at the output buffer of the decision circuit (a D-type flip-flop) at a different time, unable to be simultaneously decoded by the FEC decoder. Without loss of generality, we assume $P_{r}$ to be the slowest channel among the $n$ channels at wavelength $\lambda_{n}$. To cope with the problem of bit-skew, we introduce one additional column of D-type flip-flops before the FEC decoder and use the clock of $P_{r}$ channel to latch the $n$ D-type flip-flops. Thus, the FEC decoder has truly parallel inputs that can be simultaneously decoded. One thing to note is that the current bit of $P_{r}$ channel must arrive at the buffer before the succeeding bit of the fastest channel reaches the buffer at least by the D-type flip-flop setup-hold time $T_{\mathrm{SH}}$. This sets a limit on the system which is discussed later.

Figs. 6 and 7 show the decoder circuits for a $(15,11)$ SEC and a $(16,11)$ SEC-DED Hamming code, respectively. The syndrome generators based on "syndrome equations" [10] are similar to those parity generators of encoder circuits in Figs. 3 and 4. In the SEC decoder, when one error occurs in the received codeword, the error position is indicated by the binary syndrome, which is sent to the 4-to-16 decoder to correct the error. The correction is made simply by EXOR's. The error channel is indicated by the 4-to-16 decoder which outputs a logic high signal to invert the received error bit to the right one by the EXOR. In the SEC-DED decoder, single errors are corrected as above and passed through 3-state buffers. The extra syndrome bit $s_{o v}$ is used to detect double-error patterns when $s_{o v}=0$ and $\left(s_{4}, s_{3}, s_{2}, s_{1}\right) \neq 0$. When a double error happens, the received $k$ data channels will pass through 3 -state buffers without error correction. For two-way communications, the double-error signal can be sent back to the transmitter asking for retransmission. Here, we simply use it to prevent 


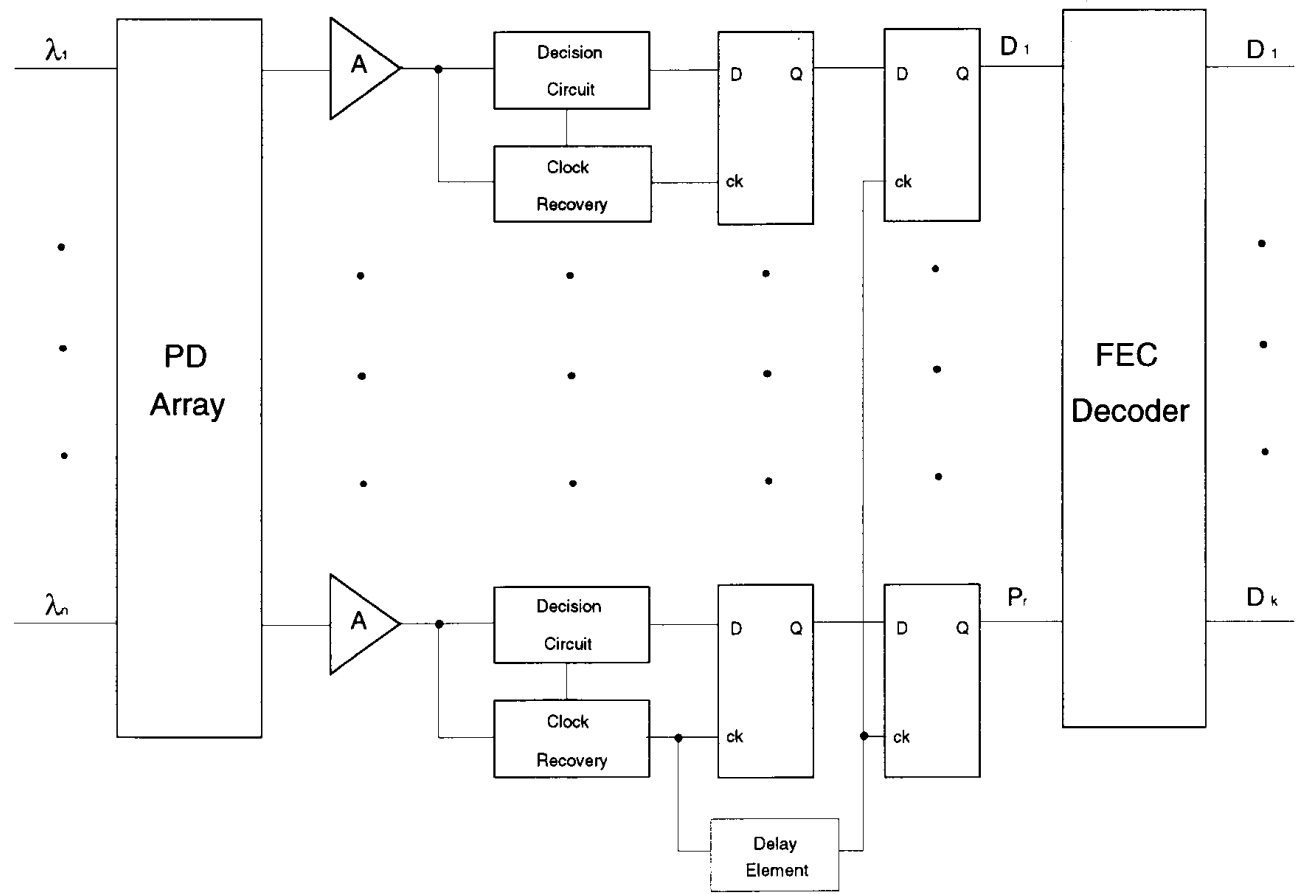

Fig. 5. Receiver architecture of a WDM coding system.

miscorrection. Again these circuits are composed of simple combinational logics which can be implemented easily by high-speed electronics.

Obviously, the delay in encoder and decoder is the gate propagation delay only. These delays can be calculated by the number of logic levels in encoder and decoder circuits, expressed as

$$
\begin{aligned}
L_{i}^{(p)} & =\left\lceil\log _{b}\left(N_{i}-1\right)\right\rceil \\
L_{i}^{(s)} & =\left\lceil\log _{b}\left(N_{i}\right)\right\rceil
\end{aligned}
$$

where $N_{i}$ is the number of "1" symbols in each row of paritycheck matrix; $L_{i}^{(p)}$ is the number of logic levels required to generate the $i$ th parity-check bit with $b$-input modulo-2 adders; $L_{i}^{(s)}$ is the number of logic levels required to generate the $i$ th syndrome bit with $b$-input modulo- 2 adders; $\lceil X\rceil$ denotes the smallest integer greater than or equal to $X$.

If all $N_{i}$ for $i=1,2, \cdots, r$ are minimum and equal (the condition of minimum equal-weight code), e.g., in the $(15,11)$ Hamming code, we can obtain the lowest propagation delay for generating parity-check and syndrome bits [11].

\section{Performance Analysis and System Limitation}

\section{A. Performance Analysis}

Hamming code is one of the "perfect" codes [10], [11], and [13] that can correct all single errors and no further doubleerror pattern. The error probability of decoded codeword $P_{w}$ can be expressed as

$$
P_{w}=\sum_{i=2}^{n}\left(\begin{array}{c}
n \\
i
\end{array}\right) P_{e}^{i}\left(1-P_{e}\right)^{n-i}
$$

where $P_{e}$ is channel BER prior to coding. Since the characteristics of LD's are different, and the attenuation and dispersion of fiber for each wavelength channel varies also, the BER of each channel is not the same and is independent. Here, for simplicity, we use the error probability of the worst channel to estimate system performance conservatively.

The BER after decoding depends on the error extension characteristics of the code. Grover analyzed the exact model of system BER with FEC [7]. The error extension of $\boldsymbol{e}$ errors occurring in a received codeword has three possible outcomes: $e+1, e, e-1$, corresponding to the increase of one additional error bit, no error extension, and fortuitously correcting one error in the primary error pattern, respectively. The average number of decoded errors resulting from $e$ primary errors for our system can be described as

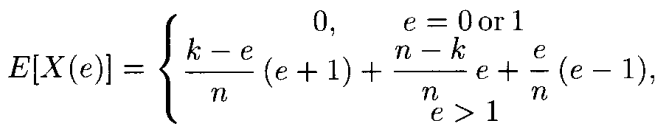

where $E[X(e)]$ is the expected number of decoded errors given $e$ primary errors in the received codeword. The decoded BER for each channel $P_{e, F E C}$ is then expressed as

$$
P_{e, \mathrm{FEC}}=\frac{1}{k} \sum_{e=2}^{n} E[X(e)] \frac{n !}{e !(n-e) !} P_{e}^{e}\left(1-P_{e}\right)^{n-e}
$$




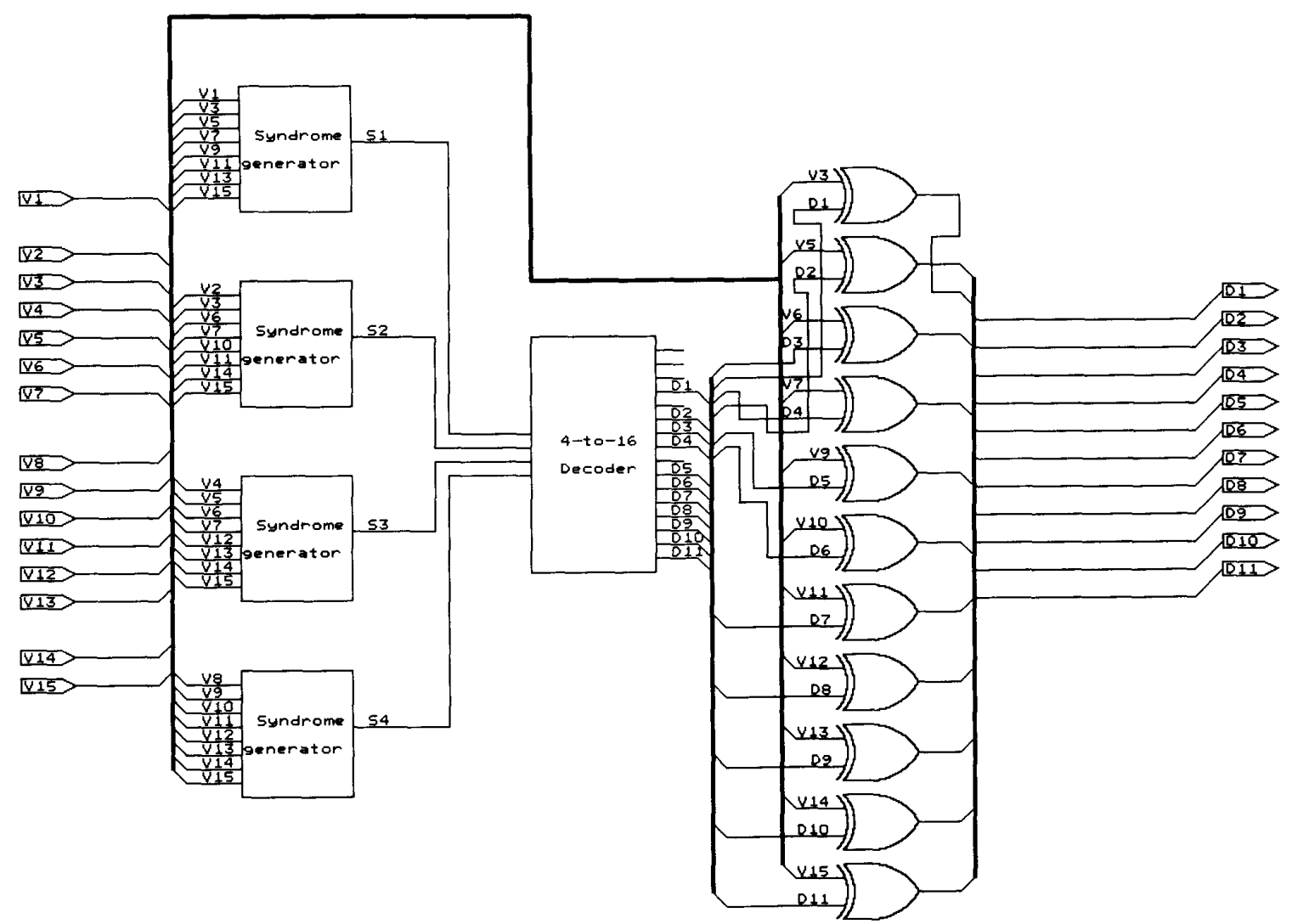

Fig. 6. Decoder circuit of a $(15,11)$ SEC Hamming code.

where $P_{e}$ is the worst uncoded BER among the channels. Fig. 8 shows the BER of three different $(n, k)$ WDM Hamming codes and the uncoded system. When $P_{e}<10^{-4}$, it can be shown that the decoded BER is dominated by the case $e=2$. In other words, FEC reduces the original BER to the order of $O\left(P_{e}^{2}\right)$. In ordinary lightwave systems with $P_{e}<10^{-9}$, FEC can approach nearly error-free transmission. For instance, the decoded BER for a $(15,11)$ WDM coding system with $P_{e}=10^{-9}$ is about equal to $3 \times 10^{-17}$. The figure also indicates that $P_{e, F E C}$ degrades as the number of channels increases. This is different from the ordinary serial Hamming coded system whose decoded BER will be improved as the encoded data number increases. In ordinary serial coding systems, system bit rate is affected by the code rate $k / n$, as the encoded data number increases, the code rate increases as well, which reduces the additional coding-induced noise. But here in the WDM coding system, the bit rate of each channel is unchanged which results in different BER performance.

FEC could provide infinite coding gain in dispersion-limited lightwave systems. Several reports concerning impacts of wavelength chirp [14], optical back reflection [15], [16], mode partition noise (MPN) [17], [18], and mode hopping of semiconductor lasers [19] in dispersive optical fiber communications have been studied and analyzed [20]. To illustrate the BER floors induced by these effects, we use the method presented by Shen and Agrawal [21] to demonstrate BER improvement in WDM coding systems. Let $S_{1}$ and $S_{0}$ be the detected signal in the $\mathrm{ON}$ and OFF states, respectively,

$$
S_{j}=\left(\frac{\eta q}{h \nu}\right)\langle M\rangle b_{j}, \quad j=0 \text { or } 1
$$

where $(\eta q / h \nu)$ is the responsivity of photodetector with quantum efficiency $\eta$ to the incident photons of energy $h \nu,\langle M\rangle$ is the average APD gain, and $b_{j}$ is the average received power. Assuming Gaussian statistics, a noise current $\sigma_{j}$ associated with the signal $S_{j}$ will result in error probability $P_{e}$ as

$$
\begin{aligned}
P_{e} & =\frac{1}{\sqrt{2 \pi}} \int_{Q}^{\infty} e^{\frac{-x^{2}}{2}} d x \\
& =\frac{1}{2} \operatorname{erfc}(Q / \sqrt{2})
\end{aligned}
$$

where erfc $(x)$ denotes the complementary error function and

$$
Q=\frac{S_{1}-S_{0}}{\sigma_{0}+\sigma_{1}}
$$

If $r$ is the $\mathrm{OFF} / \mathrm{ON}$ ratio, $r=b_{0} / b_{1}$, and $P_{\text {av }}$ is the average detected power, $P_{a v}=\left(b_{0}+b_{1}\right) / 2$, then $Q$ becomes

$$
Q=\left(\frac{\eta q}{h \nu}\right)\langle M\rangle\left(\frac{1-r}{1+r}\right) \frac{2 P_{\mathrm{av}}}{\sigma_{0}+\sigma_{1}} .
$$




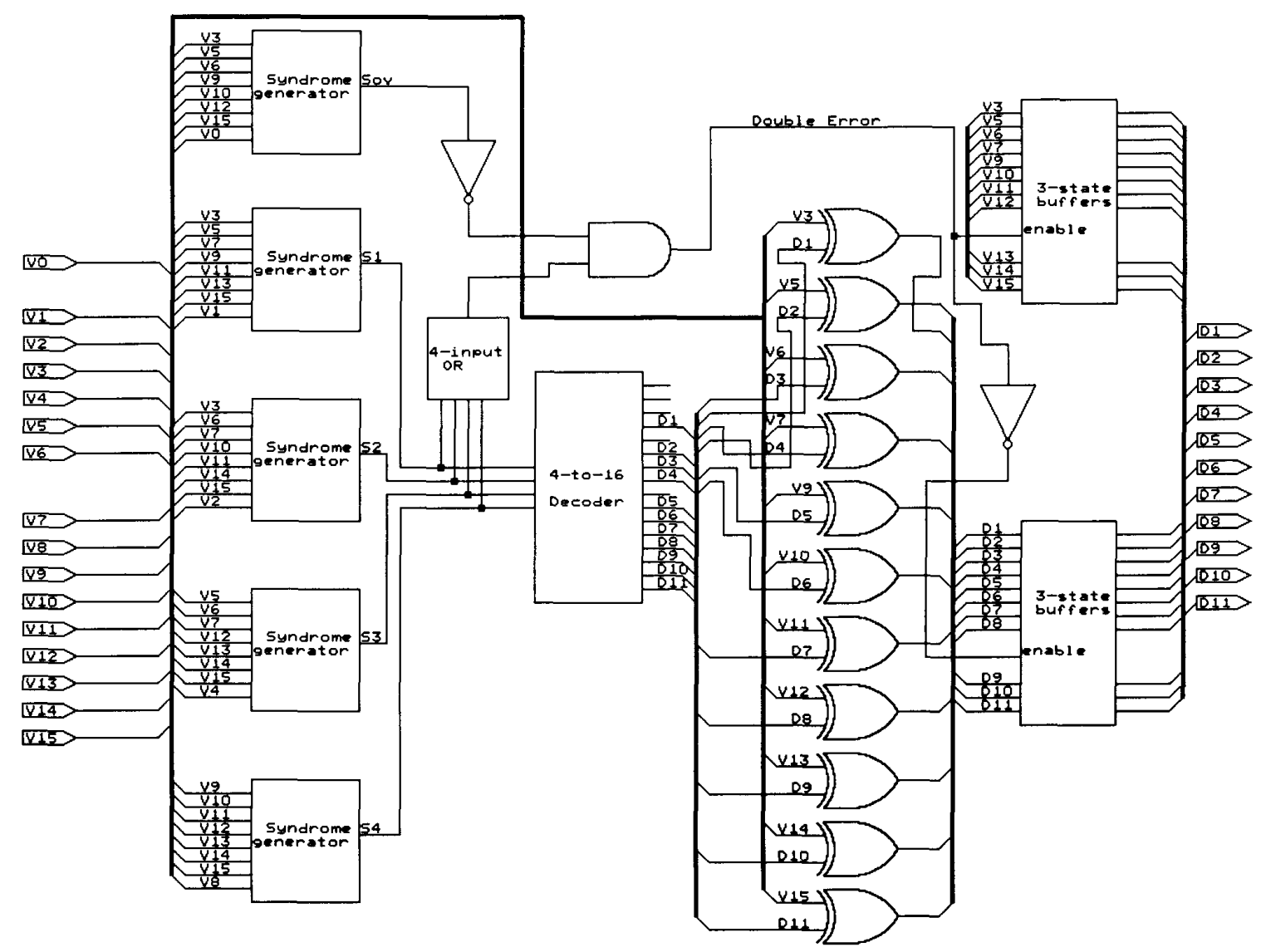

Fig. 7. Decoder circuit of a $(16,11)$ modified SEC-DED Hamming code.

The noise current $\sigma_{j}$ gets contributions from several sources and can be expressed as

$$
\begin{aligned}
\sigma_{j}^{2} & =\sigma_{c, j}^{2}+\sigma_{\mathrm{sn}, j}^{2}+\sigma_{\mathrm{sig}, j}^{2}+\sigma_{\mathrm{ref}, j}^{2}+\sigma_{\mathrm{mpn}, j}^{2}+\sigma_{\mathrm{tc}, j}^{2}, \\
j & =0 \text { or } 1
\end{aligned}
$$

where $\sigma_{c, j}^{2}, \sigma_{\mathrm{sn}, j}^{2}, \sigma_{\mathrm{sig}, j}^{2}, \sigma_{\mathrm{ref}, j}^{2}, \sigma_{\mathrm{mpn}, j}^{2}$ and $\sigma_{\mathrm{tc}, j}^{2}$ are noise currents coming from thermal noise, shot noise, laser intensity noise, reflection-induced noise, mode partition noise, and chirping-induced noise, respectively. A list of the calculation formula, the discussion and derivation of each noise source can be found in [21].

Fig. 9 shows the power of FEC in dispersion-limited lightwave systems for different $(n, k)$ Hamming code. Also given is the performance of conventional serial coding (SC) in ordinary serial transmission. In this example, those BER's for uncoded channel, SC, and WDM coding are calculated under the condition of average APD gain $\langle M\rangle=9.1$, transmission length $L=69 \mathrm{~km}$ and at the transmission rate of STS- 12 . Using (8)-(11), we can calculate and plot BER as a function of average received power. The coding gain for the WDM system at $P_{e}=10^{-9}$ is about $4 \mathrm{~dB}$, and it may have infinite coding gain if the desired bit error rate is below the error floor. Also, the error floor declines significantly after coding. This indicates that WDM coding can improve performance of a dispersion-limited lightwave system drastically. Note that the performance of SC is worse than WDM coding. To accommodate additional parity-check bits, the system bit rate of SC should be increased which will induce extra noise power. The noise bandwidth will be increased by a factor of the inverse of code rate [22]. Since the system bit rate of WDM coding is unchanged, we have

$$
B_{\mathrm{SC}}=\frac{n}{k} B_{\mathrm{WDM}}
$$

where $B_{\mathrm{SC}}$ is the noise bandwidth of SC and $B_{\mathrm{WDM}}$ is the noise bandwidth of WDM coding. Because various noises are proportional to the noise bandwidth, the BER of SC is therefore worse than that of WDM coding. This does not happen in Grover's design since they intelligently put paritycheck bits in the existing overhead fields without affecting system bit rate.

\section{B. Systems Limitation}

The major issue in parallel transmission is the bit-skew (relative shift of timing) among data channels. The bit-skew problem in a byte-wide WDM parallel transmission system has been analyzed by Loeb and Stilwell. Here we evaluate the 


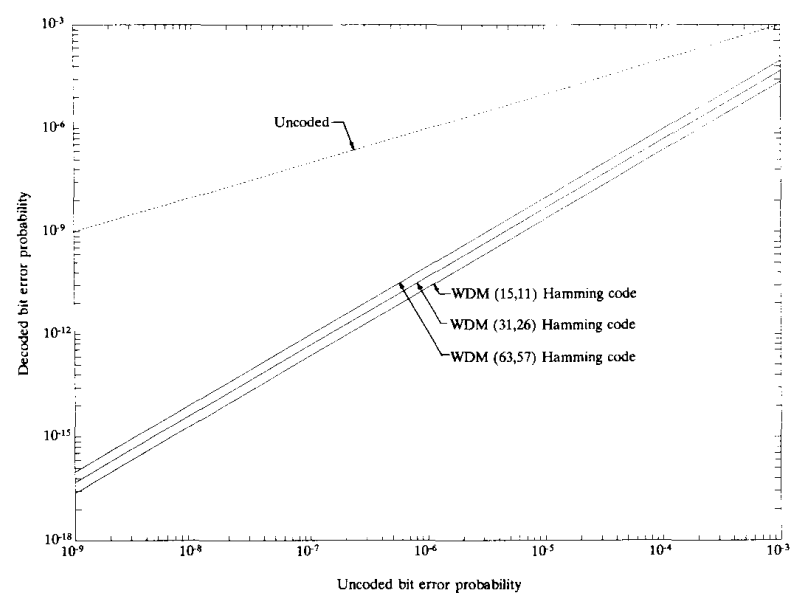

Fig. 8. Decoded BER versus uncoded BER for three different $(n, k)$ Hamming codes.

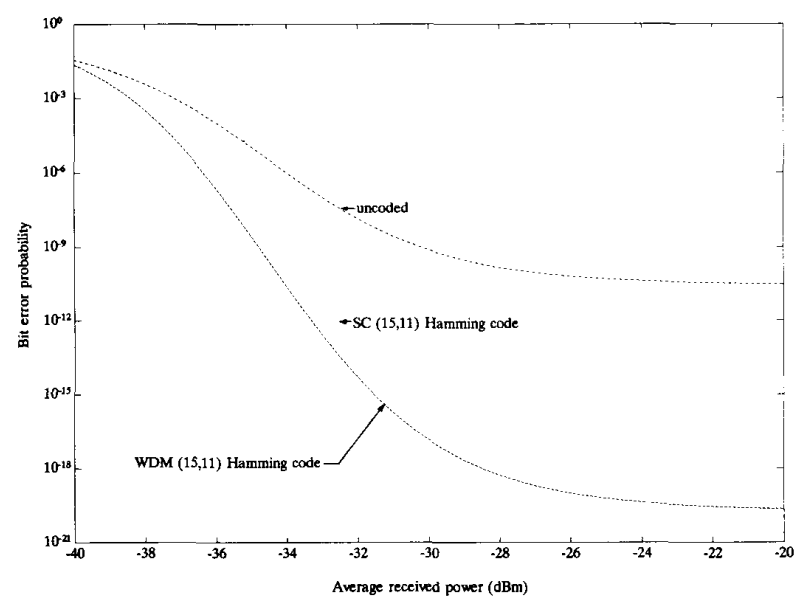

Fig. 9. Illustration of the power of WDM coding coped with BER floor for different $(n, k)$ Hamming code. SC stands for serial coding in single channel.

limitation of the WDM coding system imposed by bit-skew by considering three types of fibers.

In WDM coding systems, bit-skew happens in three components: transmitter, receiver, and fiber. The transmitter/receiver skew is due to the propagation delay difference among channels through the driver, LD array, and PD array. The skew in the fiber is caused by the group velocity mismatch among wavelength channels. The total bit skew $\Delta T_{\max }$ can be expressed as

$$
\Delta T_{\max }=\Delta T_{\mathrm{TX}}+\Delta T_{\mathrm{RX}}+\Delta T_{\mathrm{fiber}}
$$

where $\Delta T_{\mathrm{TX}}$ is the transmitter skew, $\Delta T_{\mathrm{RX}}$ is the receiver skew, and $\Delta T_{\text {fiber }}$ is the fiber skew. Usually, the fiber skew is dominant at long-distance multichannel transmission, being the main cause of time shift among channels. Hence in the following, we consider $\Delta T_{\text {fiber }}$ only [23].

The fiber skew is the maximum relative delay per unit length among channels $\Delta \tau_{\max }$, multiplied by the transmission

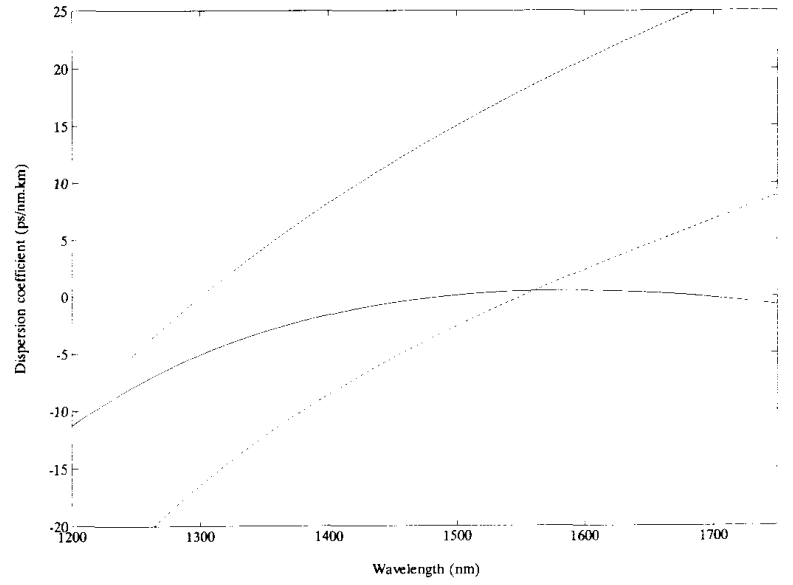

Fig. 10. Chromatic dispersion of three fibers. Dashed line: Conventional fiber. Dash-dot line: Dispersion-shifted fiber. Solid-line: Double-clad dispersion-flattened fiber.

length $L$,

$$
\Delta T_{\text {fiber }}=\Delta \tau_{\max } L .
$$

$\Delta \tau_{\max }$ is a function of the number of wavelength channels $N$ and the channel spacing $\delta$. Let $\lambda_{f}$ and $\lambda_{s}$ be wavelengths of the fastest channel and the slowest channel, respectively. The maximum relative delay per unit length is

$$
\begin{aligned}
\Delta \tau_{\max } & =\tau\left(\lambda_{s}\right)-\tau\left(\lambda_{f}\right) \\
& =\int_{\lambda_{f}}^{\lambda_{s}} D(\lambda) d \lambda
\end{aligned}
$$

where $\tau(\lambda)$ is the group delay of the fiber and $D(\lambda)$ is the chromatic dispersion coefficient. Figs. 10 and 11 illustrate the chromatic dispersion and relative group delay for three fibers: a conventional fiber (1.3- $\mu \mathrm{m}$ optimized), a dispersionshifted fiber (1.55- $\mu \mathrm{m}$ optimized), and a double-clad (DC) dispersion-flattened fiber. The fitting equations for these fibers can be found in the literature [24]-[26]. The fitting equation of conventional fibers is the three-term Sellmeier equation of the form

$$
\tau(\lambda)=A+B \lambda^{2}+C \lambda^{-2}
$$

and the fitting equation of dispersion-shifted and dispersionflatted fibers is the five-term Sellmeier equation

$$
\tau(\lambda)=A+B \lambda^{4}+C \lambda^{2}+E \lambda^{-2}+F \lambda^{-4} .
$$

From Figs. 10 and 11 we know that fastest channels for conventional and dispersion-shifted fibers are at the wavelengths of 1300 and $1550 \mathrm{~nm}$, which are the zero dispersion wavelengths of these fibers. There are two or more zerodispersion wavelengths for dispersion-flattened fibers. Fig. 10 shows that the zero-dispersion wavelengths for a doubleclad (DC) dispersion-flattened fiber are at 1500 and 1691 $\mathrm{nm}$. Note that there are two cross-points between dispersionshifted and dispersion-flattened fiber at 1528 and $1587 \mathrm{~nm}$. These cross-points show that the maximum relative delay 


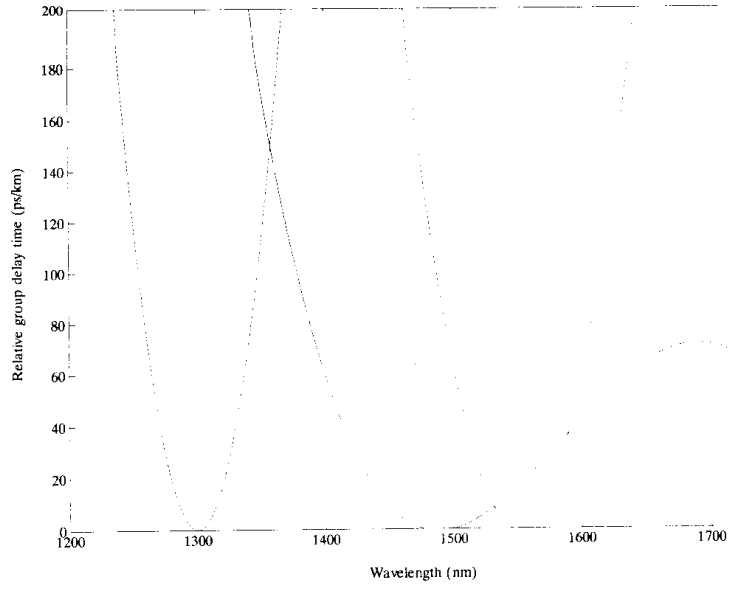

Fig. 11. Relative group delay of three fibers. Dashed line: Conventional fiber. Dash-dot line: Dispersion-shifted fiber. Solid-line: Double-clad dispersion-flattened fiber.

of dispersion-shifted fiber is smaller than dispersion-flattened fiber within these two cross-points.

With bit-skew limitation, the current bit of the slowest channel must appear at the output buffer of the decision circuit in Fig. 5 before the succeeding bit of the fastest channel by a time long enough for the D-type flip-flop to set up and hold the data. That is, we must have

$$
T-T_{\mathrm{SH}} \geq \Delta T_{\max }
$$

where $T$ is the bit duration and $T_{\mathrm{SH}}$ is setup-hold time of the D-type flip-flop. The limitation imposed by bit-skew is then

$$
T-T_{\mathrm{SH}}=\Delta \tau_{\max } L .
$$

Thus the maximum transmission distance limited by bit-skew is

$$
L=\frac{T-T_{\mathrm{SH}}}{\Delta \tau_{\max }} .
$$

Fig. 12 shows the maximum transmission distance versus channel span at STS-12 with the center channel at $1550 \mathrm{~nm}$ and $T_{\mathrm{SH}}=125 \mathrm{ps}$ [27]. The cross-point between dispersionshifted and dispersion-flattened fiber is due to the cross-points of group delay shown in Fig. 11. In the example, the maximum transmission distance of dispersion-shifted fiber is better than that of dispersion-flattened fiber for the channel span under $74 \mathrm{~nm}$. For a $30-\mathrm{nm}$ channel span, the maximum transmission distance is about $250 \mathrm{~km}$ if a dispersion-shifted fiber is used. The transmission distance of conventional fiber is severely limited by bit-skew when operating at $1.55 \mu \mathrm{m}$. However, as shown in Fig. 13, it can be much relaxed when operating at $1300 \mathrm{~nm}$.

\section{DISCUSSION}

The proposed WDM coding system is a novel approach to combine WDM with FEC. Three issues concerning comparison between serial coding and parallel WDM coding, channel survivability, and variable channel number are further discussed here.

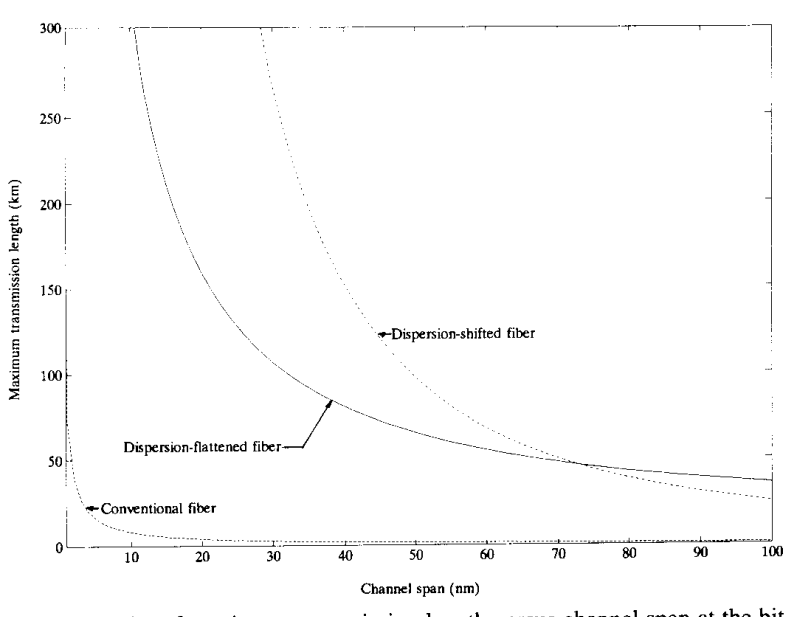

Fig. 12. Plot of maximum transmission length versus channel span at the bit rate of STS-12 with center channel at $1550 \mathrm{~nm}$.

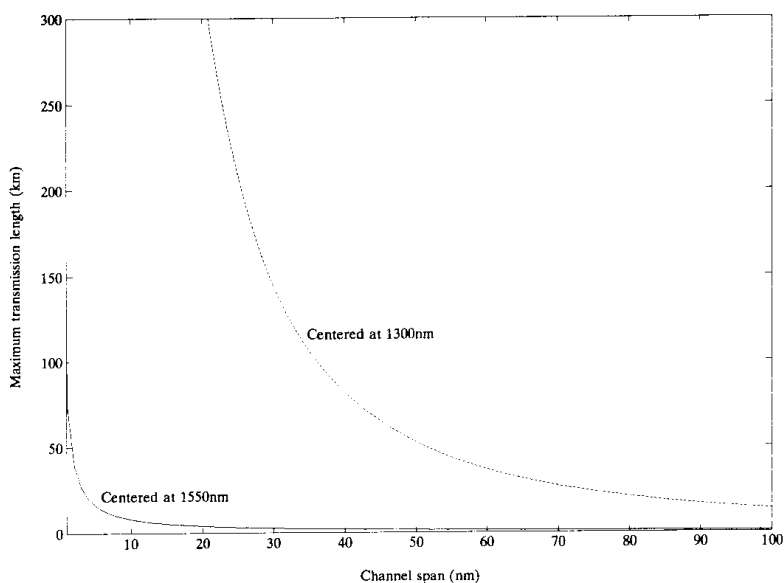

Fig. 13. Plot of maximum transmission length versus channel span for conventional fiber with center channels at $1300 \mathrm{~nm}$ and at $1550 \mathrm{~nm}$.

\section{A. Comparison between Serial Coding and Parallel WDM Coding}

Short communication links, e.g., computer and peripheral interfaces, are organized as parallel transmission because of simplicity. On the other hand, serial transmission has been adopted in long-haul high-speed data links because of physical inadequacy of multiwire or connectors and the difficulty to predict and correct bit-skew. Nevertheless, the introduction of WDM indeed opens the possibility of parallel transmission over a single-mode fiber.

Serial coding, being widely used in bandlimited channels such as satellite links, is not suitable for high-speed lightwave systems because of the need of high-speed and complex signal processing. WDM coding, being a parallel coding scheme by nature, will be a promising approach for high-capacity optical systems because of its circuit simplicity. Moreover, WDM coding does provide several interesting properties which are discussed below. 
Invariable System Bit Rate: In general, coding will change the transmission bit rate due to the addition of parity-check bits. In a serial $(n, k)$ Hamming coding system, the system bit rate will be increased by a factor $n / k$. This change of bit rate is undesirable in practical lightwave systems with a standard line rate. To avoid bit rate change, Grover and Moore [8] smartly put parity-check bits in the unassigned overhead fields of SONET frame structure to keep constant line rate. The penalty is the added complexity in signal processing which limits the encoder/decoder operating at STS-1 rate. On the other hand, WDM coding, a parallel coding approach, puts parity-check bits in additional wavelength channels without affecting the original data sequences. As a result, the system bit rate is unchanged, which is very advantageous for application in practical lightwave systems.

Simple Encoding/Decoding Processes: In a usual serial FEC, parity-check bits are added to the original data sequence. Therefore, accurate timing and modest complexity of signal processing are required to correctly put and extract paritycheck bits in the encoding/decoding processes. In Grover's design, they further put parity-check bits into unassigned overhead fields. In order to get a systematic form, they have to stagger the FEC block with the STS-1 synchronous payload envelop (SPE) to permit zero encoding delay and minimize decoding delay. In decoding, to extract and calculate the syndrome bits, they use the "pipeline" arrangement, which is a three-stage syndrome register; each stage computes part of the syndrome and completes the error indication and correction. In this approach, they have to access the SPE frame timing to replace the unassigned overhead fields with parity-check bits and to calculate the syndrome bits and then to correct the error symbol. It is the complex encoding/decoding processes and the requirement of accurate frame synchronization that make the FEC only implementable in the low-speed tributaries of a high-speed lightwave transmission link. On the contrary, WDM coding using parallel encoding/decoding processes needs only EXOR gates and D-type flip-flops without feedback connections, to be implemented easily by high-speed electronics. The simplicity of encoding/decoding processes is a direct consequence of parallel coding that puts parity-check bits in additional wavelength channels and leaves data channels unchanged. This feature makes WDM coding feasible for high-speed lightwave systems.

Number of Encoder/Decoder Pair: Lightwave transmission systems usually use a multiplexer/demultiplexer to accomplish high-speed data links. Owing to the complex signal processing needed, serial coding is implemented in each low-speed tributary to make encoding/decoding feasible with low-speed electronics [8]. Since each low-speed tributary needs an encoder/decoder pair, the total number of encoder/decoder pairs is large. For example, in Grover's design, an STS-12 line rate needs 12 STS-1 encoder/decoder pairs. Extending to WDM systems with each wavelength channel operating at STS-12 level, if we have 11 data channels, we will need $11 \times 12=132$ encoder/decoder pairs. But, with WDM coding, we just need one simple encoder/decoder being realizable easily by high-speed electronics. This saves tremendous encoder/decoder pairs, signal processing complexity, as well as signal processing time.

Frame Synchronization and Frame Format Independency: Frame synchronization is required when the information is organized in blocks. When a block code is used for forward error control, the decoder must know the boundaries between codewords in order to decode the message correctly [22]. As indicated, accurate timing and frame synchronization are needed in Grover's design since their FEC block is the whole SPE frame and they have to extract the accurate position to stuff the parity-check bits and decode them. In contrast, WDM coding is an interchannel parallel coding, the block is in the wavelength channels. Hence they do not need frame synchronization for the FEC block. Also, WDM coding is independent of the frame format of each data channel, since each bit in the data channel, whether it is in the overhead field or in the information payload of SONET frame structure, is completely irrelevant to the WDM coding.

Burst Error Correction: Generally, FEC codes used to cope with burst error need special structures, e.g., Fire code and interleaved code. A serial Hamming code cannot correct burst error. WDM coding, operating in parallel at different wavelength channels, can correct one channel burst error directly because in WDM coding, every symbol in each channel is independent of its foregoing and succeeding bits. When burst error occurs in one channel, they can be corrected directly without special coding scheme. Hence WDM coding by nature has the ability to correct one channel burst error.

Perspective: WDM or WDMA systems using direct detection and optical filters have been shown to be practical solutions for exploiting the vast bandwidth offered by optical fibers. Conventional serial FEC codes could be used in future optical systems, but as indicated, they are not very good choices for high-speed WDM systems. In contrast, WDM coding is a straightforward approach to implement FEC code. In future WDM systems, WDM coding can be used to drastically decrease the BER floor caused by the power-independent noises. The problem of WDM coding is the bit-skew due to fiber dispersion. This may be compensated by using fiber delay lines in the receiving end. Since merely the relative delay among channels is compensated, short fiber delay lines are expected. For instance, to compensate a bit-skew of $1 \mathrm{~ns}$, a fiber delay line of about $0.2 \mathrm{~m}$ is needed.

From the above discussion, we believe that WDM coding is a promising approach to reach a reliable high-capacity optical transmission.

\section{B. Channel Survivability}

One of the essences of WDM coding systems is the characteristic of interchannel coding which highly utilizes the enormous fiber bandwidth to reach a reliable communication. This is rarely seen in other telecommunication channels, e.g., satellite communications, where the bandwidth is very limited. This property makes WDM coding differ from conventional serial coding. The parallel characteristics of WDM coding also offers a special feature of channel survivability which is absent in serial coding system. 


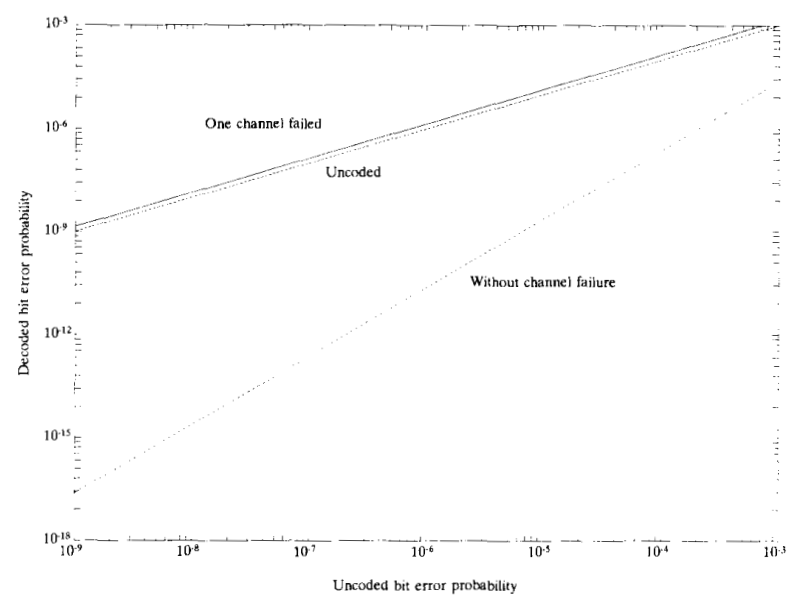

Fig. 14. Plot of decoded BER of a $(15,11)$ WDM Hamming code with one channel failure. Solid line: Decoded BER with one channel failure. Dashed line: Uncoded BER. Dash-dot line: Decoded BER without channel failure.

Here we analyze the decoded BER when there is one channel failed. When a channel failed, the uncoded BER of the failed channel is assumed to be $1 / 2$. After decoding, since most of the errors will be corrected, the decoded BER will be very small. The decoded codeword error rate is

$$
\begin{aligned}
P_{w, \text { fail }}= & \frac{1}{2} \sum_{j=1}^{n-1}\left(\begin{array}{c}
n-1 \\
j
\end{array}\right) P_{\mathrm{e}}^{j}\left(1-P_{e}\right)^{n-j-1} \\
& +\frac{1}{2} \sum_{k=2}^{n-1}\left(\begin{array}{c}
n-1 \\
k
\end{array}\right) P_{e}^{k}\left(1-P_{e}\right)^{n-k-1}
\end{aligned}
$$

where the first and second terms account for cases where the failed channel happens to be wrong or correct, respectively. The decoded BER is somewhat more complicated to derive. An approximation can be used to estimate the system performance as [13]

$$
P_{e, \mathrm{FEC}} \approx \frac{d_{\mathrm{min}}}{n} P_{w, \text { fail }}
$$

where $d_{\min }$ is the minimum Hamming distance, which is 3 in the Hamming code. The decoded BER for each channel with one channel failure is shown in Fig. 14 for a $(15,11)$ WDM Hamming code. The figure reveals that the decoded BER with one channel failure is just slightly worse than the uncoded BER without channel failure, meaning that the failed channel after FEC decoder can have nearly the same BER as an uncoded channel. Thus WDM coding systems can provide channel survivability for one channel failure.

\section{Variable Channel Number and Shortened Hamming Code}

In WDM systems, channel number may be varied--we may need a flexible codeword length for WDM coding system. Fortunately, Hamming code can provide shortened codeword format. For instance, we can construct a $(12,8)$ shortened Hamming code for a system with 8 data channels. In this case, the code rate, $k / n$ is reduced.
The shortened Hamming code is obtained by deleting $l$ columns from the parity-check matrix $\boldsymbol{H}$ of a Hamming code. This deletion results in an $r \times\left(2^{r}-l-1\right)$ matrix $\boldsymbol{H}_{\boldsymbol{s}}$. Using $\boldsymbol{H}_{\boldsymbol{s}}$ as the shortened parity-check matrix, we obtain a shortened Hamming code with the following parameters:

number of parities, $r: r=n_{s}-k$

block length of codeword, $n_{s}: n_{s}=2^{r}-l-1$

data length, $k: k=n_{s}-r-l$

minimum distance, $d_{\min }: d_{\min } \geq 3$.

If we delete columns from $\boldsymbol{H}$ properly, we may obtain a shortened Hamming code with minimum distance 4 . We can also obtain a shortened distance- 4 Hamming code by deleting any $l$ columns from the $\boldsymbol{H}^{\prime}$ matrix. However, for convenience in encoding and decoding logic, it is better to delete the rightmost $l$ columns of the parity-check matrix [10], [11]. The average decoded error of a shortened Hamming code as [8] is given below.

$$
E[X(e)]=\left\{\begin{array}{c}
0, \quad e=0 \text { or } 1 \\
\frac{k-e}{n_{\max }}(e+1)+\frac{n_{\max }-k}{n_{\max }} e+\frac{e}{n_{\max }}(e-1),
\end{array}\right.
$$

where $E[X(e)]$ is the expected number of decoded errors given $\boldsymbol{e}$ primary errors in the received codeword $n_{\max }\left(n_{\max }=\right.$ $2^{r}-1$ ) is the maximum block size of Hamming code, and $P_{e, \mathrm{FEC}}$ is then expressed as before.

\section{CONCLuSIONS}

Serial FEC codes are rarely used in high-speed lightwave systems because of change of system bit rate, complicated encoding/decoding processes, and small coding gain in powerlimited lightwave systems. However, a single-mode fiber can provide enormous bandwidth to carry high-capacity information via WDM techniques and, according to Shannon's theorem, this abundant bandwidth can be utilized to implement parallel FEC codes.

Here we have surveyed a WDM coding scheme to realize reliable high-capacity WDM systems by employing the abundant fiber bandwidth for coding purpose. The key idea of WDM coding is using the tremendous fiber bandwidth to carry high-capacity information and to perform interchannel FEC coding. The interchannel coding is a parallel process which is different from usual serial codings. Specifically, the WDM coding system could offer unaltered data rate, rendering it very suitable for standard lightwave systems; a simple encoding/decoding process makes it be easily realized by high-speed electronics; and there is a tremendous saving of encoder/decoder pairs. In addition, it can provide medium or infinite coding gain as well as to correct one channel burst error.

Indeed, WDM coding is a good combination of electrical and optical techniques to reach a reliable high-capacity longhaul lightwave system. In the electrical domain, we use an interchannel coding scheme with simple encoding/decoding, which is easily implemented by high-speed electronics. In the optical domain, we use the enormous fiber bandwidth to carry high-capacity information as well as to carry parity-check 
channels. The resultant system is superior to the usual serial coding systems and is a promising approach toward a reliable long-haul WDM system.

\section{REFERENCES}

[1] Yu and T. S. Francis, Optics and Information Theory. New York: Wiley, 1984

[2] R. E. Ziemer and W. H. Tranter, Principles of Communications, New York: Mifflin, 2nd ed., 1985

[3] L. Pierre, Fundamental Concepts in Communication, Englewood Cliffs, NJ: Prentice-Hall, 1990.

[4] J. Wu, C. Wang, and I. Wu, "Coding to relax laser linewidth requirements and improve receiver sensitivity for coherent optical BPSK communications," J. Lightwave Technol., vol. 8, no. 4, pp. 545-553, Apr. 1990.

[5] L. J. Cimini, Jr., and G. J. Foschini, "Coding of on-off keying signals impaired by phase noise," IEEE Trans. Commun., vol. 38, no. 9, pp. 1301-1307, Sept. 1990.

[6] G. J. Foschini and A. A. M. Saleh, "Overcoming optical amplifier intermodulation distortion using coding in multichannel communications systems," IEEE Trans. Commun., vol. 38, no. 2, pp. 187-191, Feb. 1990.

[7] W. D. Grover, "Forward error correction in dispersion-limited lightwave systems," J. Lightwave Technol., vol. 6, no. 5, pp. 643-654, May 1988.

[8] W. D. Grover and T. E. Moore, "Design and characterization of an errorcorrecting code for the SONET STS-1 tributary," IEEE Trans. Commun. vol. 38 , no. 4 , pp. $467-476$, Apr. 1990

[9] H. Kobrinski et al., "Demonstration of high capacity in the LAMBDANET architecture: A multiwavelength optical network," Electron Lett., vol. 23, no. 16, pp. 824-826, July 1987.

[10] T. R. N. Rao and E. Fujiwara, Error-Control Coding for Computer Systems. Englewood Cliffs, NJ: Prentice-Hall, 1989.

[11] S. Lin and D. J. Costello, Jr., Error Control Coding: Fundamentals and Applications. Englewood Cliffs, NJ: Prentice-Hall, 1983.

[12] M. L. Loeb and G. R. Stilwell, Jr., "High-speed data transmission on an optical fiber using a byte-wide WDM system," IEEE J. Lightwave Technol., vol. 6, no. 8, pp. 1306-1311, Aug. 1988.

[13] G. C. Clark, Jr., and J. B. Cain, Error-Correction Coding for Digital Communications. New York: Wiley, 1985.

[14] S. Yamamoto, M. Kuwazuru, H. Wakabayashi, and Y. Iwamoto, "Analysis of chirp power penalty in 1.55- $\mu \mathrm{m}$ DFB-LD high-speed optical fibe transmission systems," IEEE J. Lightwave Technol., vol. LT-5, no. 10, pp. 1518-1524, Oct. 1987.

[15] G. P. Agrawal and T. M. Shen, "Effect of fiber-far-end reflections on the bit error rate in optical communication with single-frequency semiconductor lasers," J. Lightwave Technol., vol. 4, no. 1, pp. 58-63, Jan. 1986.

[16] B. R. Clarke, "The effect of reflections on the system performance of intensity modulated laser diodes," J. Lightwave Technol., vol. 9, no. 6, pp. 741-749, June 1991.

[17] J. C. Cartledge, "Performance implications of mode partition fluctuation in nearly single longitudinal mode lasers," J. Lightwave Technol., vol 6 , no. 5, pp. 626-635, May 1988.

[18] R. H. Wentworth, G. E. Bodeep, and T. E. Darcie, "Laser mode partition noise in lightwave systems using dispersive optical fiber," J. Lightwave Technol., vol. 10, no. 1, pp. 84-89, Jan. 1992.

[19] M. Ohtsu and Y. Teramachi, "Analysis of mode partition and mode hopping in semiconductor lasers," IEEE J. Quantum Electron., vol. 25 , no. 1, pp. 31-38, Jan. 1989.

[20] R. Heidemann, "Investigations on the dominant dispersion penalties occurring in multi-gigabit direct detection systems," J. Lightwave Technol., vol. 6, no. 11, pp. 1693-1697, Nov. 1988.

[21] T. M. Shen and G. P. Agrawal, "Computer simulation and noise analysis of the system performance of $1.55-\mu \mathrm{m}$ single-frequency semiconductor lasers," J. Lightwave Technol., vol. LT-5, no. 5, pp. 653-659, May 1987.

[22] B. Sklar, Digital Communications-Fundamentals and Applications. Englewood Cliffs, NJ: Prentice-Hall, 1988

[23] K. Kaede et al., "12-channel parallel optical-fiber transmission using a low-drive current $1.3-\mu \mathrm{m}$ LED array and a p-i-n PD array," J. Lightwave Technol., vol. 8, no. 6, pp. 883-888, June 1990.

[24] G. Keiser, Optical Fiber Communications. Singapore: McGraw-Hill, 1991.

[25] Y. Miyajima, T. Kawata, and Y. Negishi, "Considerations on group delay fitting equations for dispersion-shifted fibers," Electron. Lett., vol. 23, no. 25 , pp. $1381-1383$, Dec. 1987.

[26] L. G. Cohen, "Comparison of single-mode filter dispersion measurement techniques," J. Lightwave Technol., vol. LT-3, no, 5, pp. 958-966. Oct. 1985.

[27] M. Madihian et al., "Application of AlGaAs/GaAs HBT's to high-speed CML logic family fabrication," IEEE Trans. Electron Devices, vol, 36, no. 4, pp. 625-631, Apr. 1989.

S.-K. Shao, photograph and biography not available at the time of publication.

M.-S. Kao, photograph and biography not available at the time of publication. 